\title{
Radiologic and Pathologic Findings of a Huge Solitary Fibrous Tumor of the Liver with Malignant Transformation: A Case Report
}

\author{
Niloofar Ayoobi Yazdi ${ }^{1,2}$, Habibollah Dashti ${ }^{1,3}$, Masoomeh Safaei ${ }^{4}$, Faezeh Salahshour ${ }^{1}$, Maryam \\ Fotouhi $^{5}$, Ali Jafarian ${ }^{1,3}$ and Ghazaleh Amjad ${ }^{6,{ }^{*}}$ \\ ${ }^{1}$ Liver Transplantation Research Center, Imam Khomeini Hospital Complex, Tehran University of Medical Sciences, Tehran, Iran \\ ${ }^{2}$ Department of Radiology, Imam Khomeini Hospital Complex, Tehran University of Medical Sciences, Tehran, Iran \\ ${ }^{3}$ Department of Hepatopancereatobiliary \& Liver Transplantation, Imam Khomeini Hospital Complex, Tehran University of Medical Sciences, Tehran, Iran \\ ${ }^{4}$ Department of Pathology, Cancer Institute, Imam Khomeini Hospital Complex, Tehran University of Medical Science, Tehran, Iran \\ ${ }^{5}$ Quantitative MR Imaging and Spectroscopy Group, Research Center for Cellular and Molecular Imaging, Tehran University of Medical Sciences, Tehran, Iran \\ ${ }^{6}$ Department of Radiology, Shahid Akbar Abadi Hospital, Iran University of Medical Sciences, Tehran, Iran \\ "Corresponding author: Department of Radiology, Shahid Akbar Abadi Hospital, Iran University of Medical Sciences, Tehran, Iran. Email: Ghazal_1986_amjad@yahoo.com
}

Received 2018 March 09; Revised 2019 November 05; Accepted 2019 November 12.

\begin{abstract}
Solitary fibrous tumors (SFTs) are mesenchymal tumors that mostly occur in the pleural cavity. Extra-thoracic location is rare and hepatic origin is extremely rare. Most lesions are benign, 10\% - 15\% show aggressive behavior and few metastasizing SFTs have been reported. Imaging features of solitary fibrous tumors of the liver (SFTLs) are nonspecific and definite diagnosis usually needs histopathological and immunohistochemistry evaluation. We report ultrasound, CT and MRI features of such a rare malignant SFTL in a 47-year-old man who came with vague abdominal symptoms in detail along with reviewing literature considering imaging features which is valuable for radiologists. The lesion seen as a huge dominantly cystic lesion on ultrasound was initially misinterpreted as hydatid. On CT scan it was seen as a large encapsulated mass with arterial hyper-enhancement and delayed contrast retention and multiple cystic spaces. On MRI, solid components showed iso-intensity to adjacent liver on T1 and T2 images, small areas of restriction on diffusion weighted imaging (DWI) and few hemorrhagic cystic components beside enhancement pattern and multiple large cystic components similar to CT scan. Our patient was admitted for resection of huge hepatic mass and experienced an episode of altered mental status due to hypoglycemia during hospital admission, which is a rare finding in SFTL. The patient underwent right hepatectomy and solitary fibrous tumor was confirmed on pathologic examination of the resected tumor. Hypoglycemic episodes were resolved and the patient was asymptomatic in 28 months follow-up.
\end{abstract}

Keywords: Solitary Fibrous Tumor, Enhanced MRI, CT Scan, Malignant Transformation

\section{Introduction}

Solitary fibrous tumors (SFTs) are mesenchymal neoplasms which rarely grow in the liver. These tumors were first identified by Klemperer and Robin in 1931. They reported that the origin of these tumors are more frequently serosal surfaces such as the pleura (1). Nonetheless, in later studies, it appeared that these tumors can also grow in other areas such as meninges, spinal cord, peritoneum, retroperitoneum, adrenal gland, thyroid gland, pelvis, soft tissue, orbit, and mediastinum (2). Liver has been rarely reported as the location of solitary fibrous tumor and there are less than 100 cases in the literature addressing it (3).

Most solitary fibrous tumors of the liver (SFTL) are detected incidentally because they are frequently asymptomatic despite their remarkable size. Patients may have presentations including hypoglycemia or signs and symp- toms of the mass effect on adjacent organs in the abdomen, pelvis, and thorax (4). Hypoglycemia happens as the results of overproduction of high molecular weight insulinlike growth factor II (5).

The radiologic findings of SFTLs are non-specific and the final diagnosis needs histopathological and immunohistochemical investigation. Here, we report the radiological imaging findings and the differential diagnosis of a newly diagnosed patient with SFTL.

\section{Case Presentation}

A 47-year-old male presented with fullness and right upper quadrant (RUQ) abdominal pain since 2 months ago. Abdominal ultrasound was done in another center and a large multiloculated solid cystic lesion in the right liver lobe was found (Figure 1). Initially hydatid cyst was 
considered as the most probable diagnosis but the serology test was negative. Then, the patient was referred to our hepatobiliary center and he underwent tri-phasic contrast-enhanced abdominopelvic computed tomography (CT) scan before and 40, 70, and 180 seconds after injection of 120 cc of Omnipaque(Iohexol) 350 with $1.5 \mathrm{~mm}$ collimation and $3 \mathrm{~mm}$ slice thickness with emotion 16 Siemens CT scanner. CT scan demonstrated a mass measuring about $325 \times 190 \times 186 \mathrm{~mm}$ containing multiple cysts in the right liver lobe. The tumor revealed arterial enhancement in the solid part and retained contrast in the delayed phase. It had significant mass effect on the hepatic portion of inferior vena cava (IVC) (decreased anteroposterior diameter to about $5 \mathrm{~mm}$ ) without obvious evidence of invasion. The tumor had extensive adhesions to the right hepatic vein. There were also disposition of the second portion of the duodenum and head of the pancreas due to tumor mass effects (Figure 2).

In complementary magnetic resonance imaging (MRI) study, which was performed with Siemens Avanto fit MR machine, in axial $\mathrm{T} 1$ in-phase and opposed-phase [repetition time(TR): $240 \mathrm{~ms}$, echo time(TE): 2 ms and 5 slice thickness (ST): 6], axial and coronal HASTE T2 weighted (TR: 900 ms, TE: 82 ms, slice thickness: 6 and field-of-view [FOV]: 380 $\times 321)$ and diffusion weighted imaging (DWI) with b value of 0,500,1000 $\mathrm{mm}^{2} / \mathrm{s}$ (TR: $4000 \mathrm{~ms}$, TE: $56 \mathrm{~ms}$ and ST: 6), apparent diffusion coefficient (ADC) map and dynamic contrast enhanced volumetric interpolated breath-hold exam-

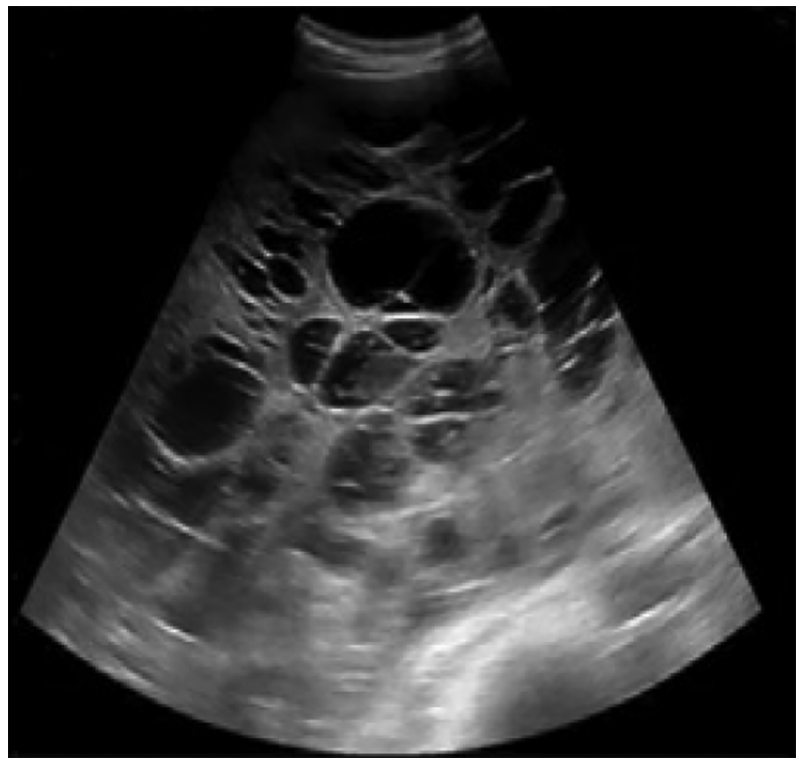

Figure 1. A 47-year-old man with 2 months fullness and right upper quadrant abdominal pain. Ultrasonography (US) exam of the right liver lobe demonstrates a bulky solid cystic mass. ination (VIBE) images (TR: $7 \mathrm{~ms}$, TE: $3 \mathrm{~ms}$, ST: 8 flip angle(FA): 20 and FOV: $400 \times 400$ ) a mass with heterogeneous signal intensity was seen in the right liver lobe. The solid components demonstrated iso-signal in $\mathrm{T} 1$ and $\mathrm{T} 2$ sequences in comparison to normal parenchyma. The cystic parts determined mostly hypo-intensity signal in T1 but had some foci of high signal due to hemorrhage. These portions had high signal in T2 sequence. In DWI MRI, small areas of solid parts showed diffusion restriction with minimum ADC value of approximately $0.55-0.85 \times 10^{-3} \mathrm{~mm}^{2} / \mathrm{s}$. After gadolinium administration, late arterial phase showed avid enhancement along with retained enhancement in the delayed phase (Figure 3).

Lab tests revealed normal liver function and erythrocyte sedimentation rate (ESR) level. Tumor markers were negative. The patient had an episode of altered mental status during admission. Further evaluations revealed hypoglycemia with blood glucose levels of $23 \mathrm{mg} / \mathrm{dL}$.

Based on the clinical presentation, lab tests, CT and MRI findings, the preliminary radiological differential diagnoses included hyper-vascular metastatic lesions such as neuroendocrine tumor or gastrointestinal stromal tumor (GIST), hepatocellular adenoma, mesenchymal tumors such as leiomyoma and PEComa (perivascular epithelioid cell neoplasm). Hepatocellular carcinoma and fibro-lamellar carcinoma were less probable considering large cystic components, absence of washout and absence of scar or calcification, respectively. Octreotide scan was recommended as a complementary evaluation which was actually negative then the biopsy was conducted with intercostal approach and histopathology study was suggestive of solitary fibrous tumor of the liver (SFTL). Ultimately right liver lobectomy was performed.

On gross examination, the large right liver lobe mass measured $30 \times 21 \times 17 \mathrm{~cm}$. The external surface was brownish and smooth. The liver was covered by capsule, but it was ruptured in some foci. Further sections revealed a creamy-brown well-delineated mass with multicystic degeneration (Figure 4).

In microscopic examination, liver tissue was infiltrated by a plump of spindle cell tumor. Tumor had hypoand hyper-cellular areas with foci of necrosis and peritheliomatous growth. Mitotic activity was up to $4 \mathrm{mf} / 10$ HPF. Focally, the neoplastic cells showed marked pleomorphism. Tumor cells were positive for vimentin, CD34, MIC2 and Bcl-2. epithelial membrane antigen (EMA), cytokeratin, desmin, smooth muscle actin (SMA), CD57, S100, CD31 and collagen IV were negative. KI67 was measured 3\% - 5\% (Figure 4).

The final histological and immunohistochemistry (IHC) analysis exposed solitary fibrous tumor with cystic degeneration and foci highly suggestive for malignant 

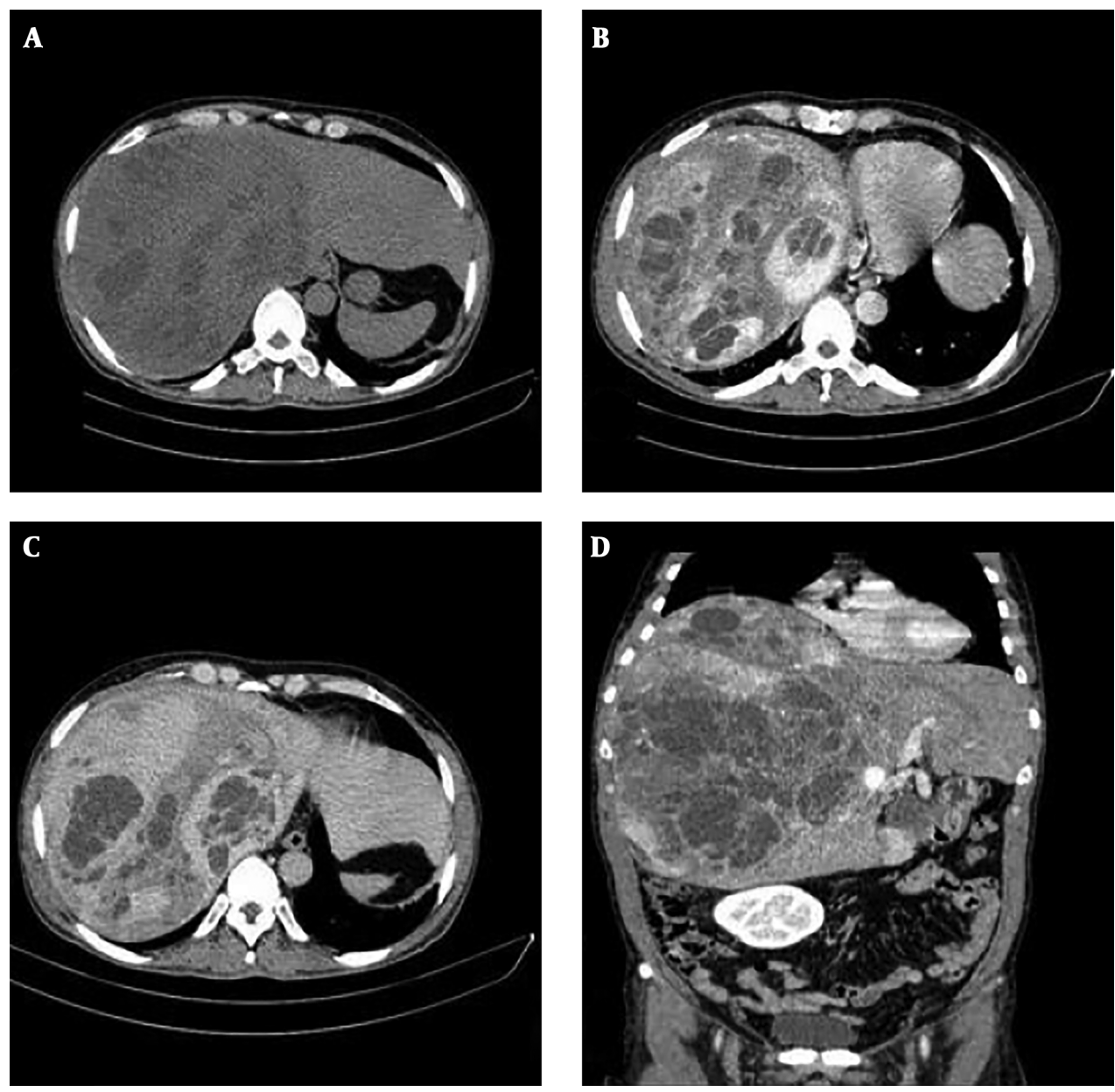

Figure 2. Non contrast enhanced CT scan (A) and contrast enhanced CT scan (B-D) of the liver. A large heterogeneous mass with cystic components is seen in the right liver lobe (A). Axial contrast CT scan shows large solid cystic mass in the right liver lobe (B). It reveals avid enhancement of the solid part in the arterial phase (C). Solid portions retained the contrast in delayed phase $(\mathrm{D})$.

transformation. At follow-up 28 months after surgical intervention, the patient was asymptomatic with no signs of tumor recurrence or metastases.

\section{Discussion}

SFTs are mesenchymal neoplasms previously expressed as hemangiopericytoma that classically origin from the pleura, but they have been reported in other extra-thoracic sites $(1,2)$. Liver is an extremely rare origin of SFTs with only 84 cases reported in the English literature since 1958 (3). These tumors are more common in females. Patients' average age at diagnosis is 61 years (6).

The clinical presentations in hepatic SFTs vary from asymptomatic to nausea, vomiting, abdominal fullness, abdominal pain, weight loss, and jaundice (7). It also might present with sign and symptoms of mass effect on adjacent visceral or neurovascular structures. There are reports of incidental findings in abdominal imaging studies $(8,9)$.

Imaging features of hepatic SFTs have been nonspecific and various. In sonography, they could be either hypo- or hyperechoic with or without calcifications (3). Frequently, they present as a heterogeneous solid welldelineated ovoid mass but sometimes, cystic component can be seen in the tumor with few reports of cystic types $(10,11)$. Echogenicity is usually more than the normal liver. Internal vascularity with occasional large vessels could be perceived in the tumor by Doppler ultrasonography (US) (12).

CT scan with intravenous contrast is commonly used for better tumor evaluation. In CT scan, hepatic SFTs could be presented as heterogeneous, hypodense, and 

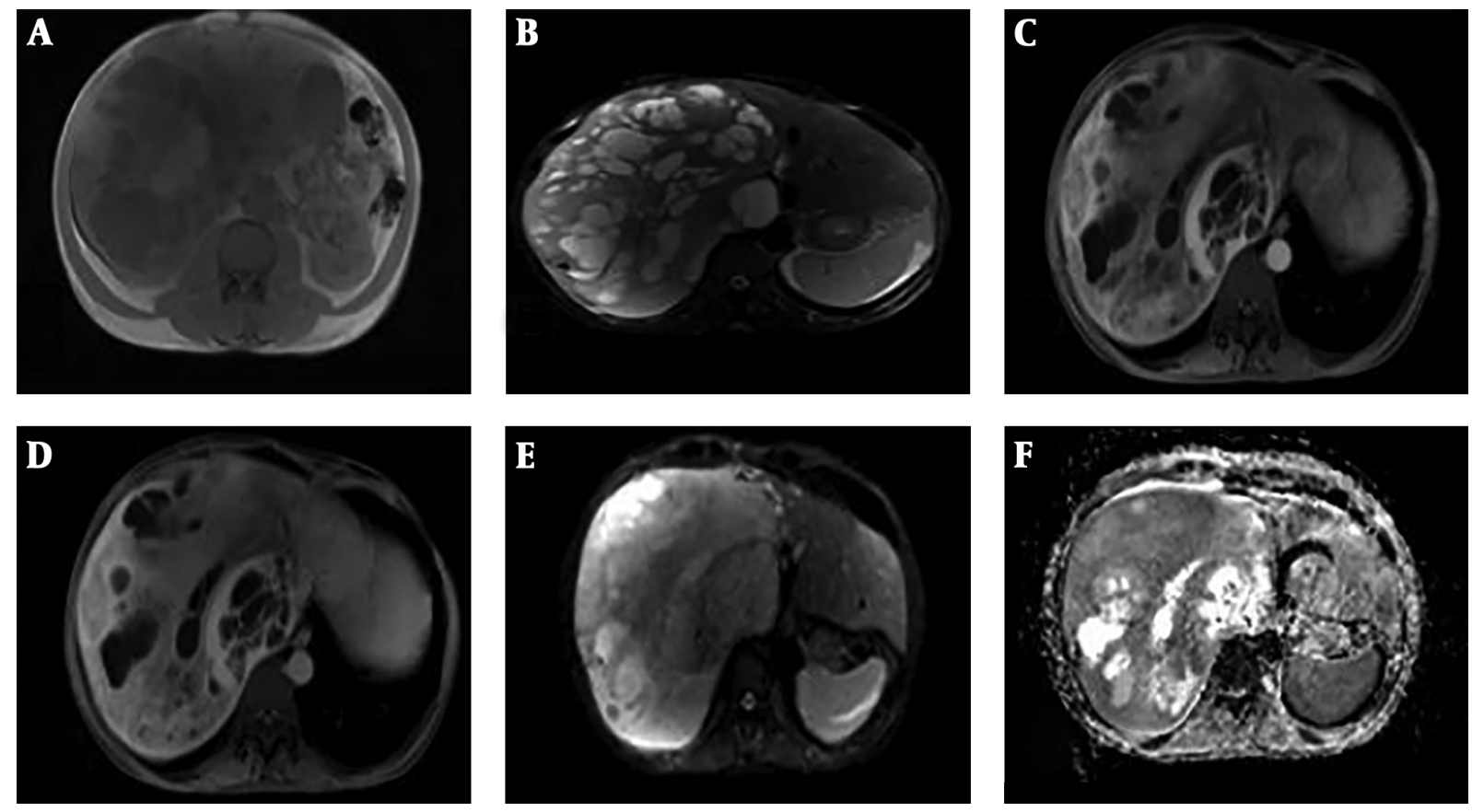

Figure 3. Huge solid cystic mass is seen in the right liver lobe. In axial T1, solid components demonstrate iso-signal intensity in comparison to normal parenchyma. Foci of high-signal intensity is also seen due to hemorrhage (A). In axial T2 fat saturation sequence, solid components are iso-signal with normal parenchyma (B). Solid parts reveal avid enhancement in the late arterial phase (C) that retain contrast material in the delayed phase (D). Some part of solid component show diffusion restriction (E and F).

well-defined mass. They usually show contrast enhancement during early arterial phase due to their hypervascularity. This enhancement may be surrounded by a thick capsule (11-16). Different degrees of enhancement associated with multiple hypodense areas may be recognized in the portal phase (1 minute). The enhancement progresses during venous and delayed phases ( 5 minute)(11) but washout in delayed phase is also reported in some studies (13, 16).

In addition, there have been reports of hypodensity due to intratumoral necrosis and calcification within a mass or in the capsule $(10,12)$. The tumor might show displacement effects on adjacent vessels. It could also compress the bile ducts (11).

Findings of abdominal MRI are habitually correspondent to abdominal CT scan $(11,12)$.

The SFTs present as heterogeneous hypo-intense or iso-intense mass compared to normal parenchyma in T1weighted and hypo-intense, iso-intense or hyper-intense mass compared to the liver parenchyma in T2-weighted sequences (11). Besides, hyper intensity due to cystic degeneration or necrosis could be observed (10). After injection of gadolinium, progressive enhancement from the center to the periphery starting in the arterial phase and staying in the venous and delayed phases due to high collagen con- tent were noted (11).

Some studies have reported the ADC map extracted from DWI could be useful to differentiate between malignant and benign lesions, monitor and quantify their extension suggesting the malignant lesion ADC value is lower than a benign lesion owing to higher cellular intensity. In a study about SFT of the pleura, the value range of malignant lesion has reported $1.2-1.9 \times 10^{-3} \mathrm{~mm}^{2} / \mathrm{s}$. Our results show minimum ADC value about $0.55-0.85 \times 10^{-3} \mathrm{~mm}^{2} /$ sand it is correlated with the pathological reports as high mitotic activity [> 4/10 high-power fields (HPF)], cytological atypia and hypercellularity (17).

Efficacy of more advanced imaging modality such as positron emission tomography (PET) in identification of SFTs is still controversial. Perhaps their utility in diagnosis of distant metastasis is more reasonable. SFTLs show higher radiotracer uptake in comparison to normal liver parenchyma in PET evaluation (10).

Radiologic findings cannot differentiate between SFTLs and other hypervascular hepatic lesions such as metastatic or primary neuroendocrine tumor (probably insulinoma due to its hyper vascularity and history of hypoglycemia), fibrolamellar carcinoma or hepatocellular carcinoma, other hypervascular hepatic metastasis (GIST) or other mesenchymal tumors such as fibrosarcoma, 

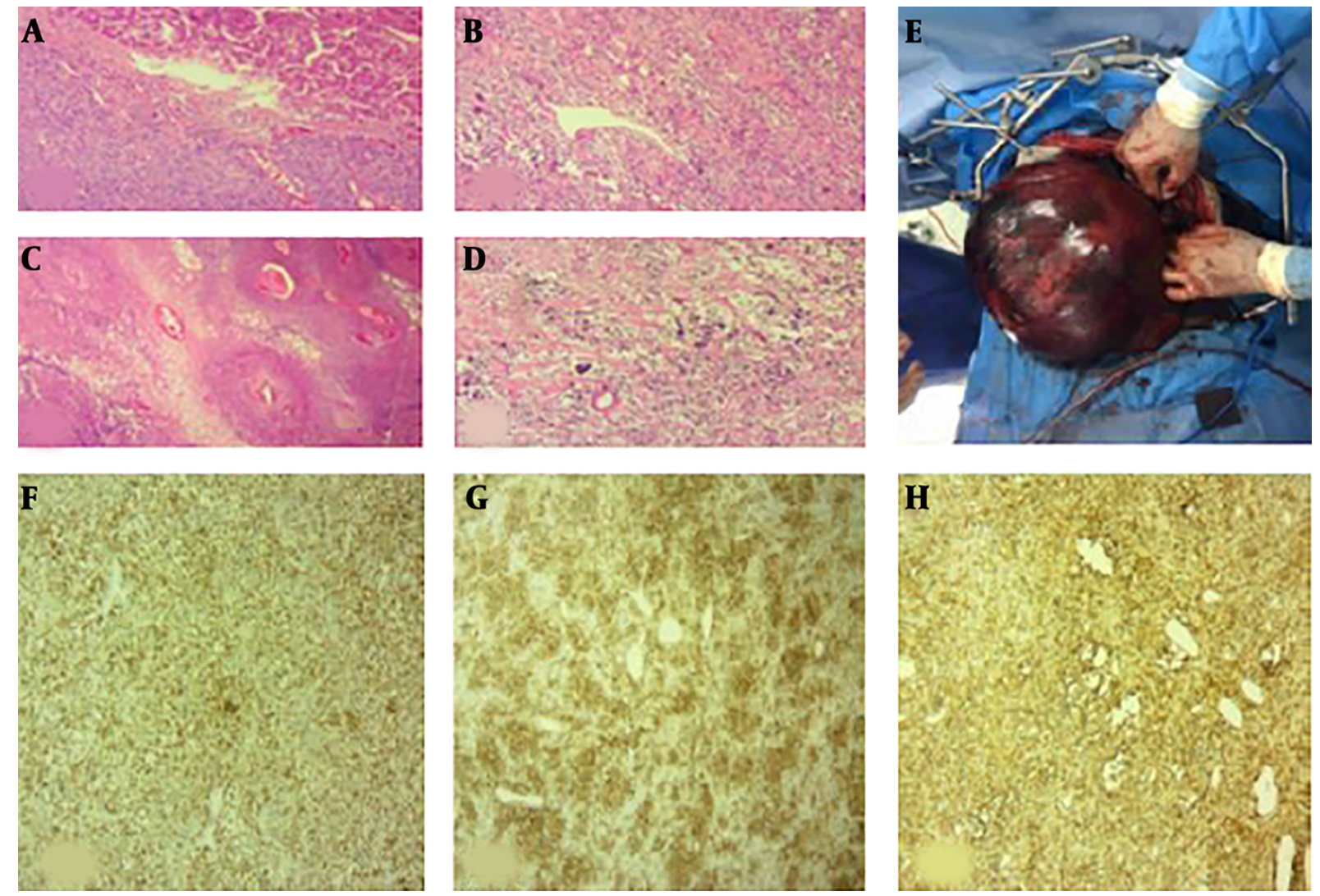

Figure 4. Microscopic examination of the lesion (A - D); Well defined neoplasm in liver (A) that has been arranged in hypo- and hyper-cellular areas, collagen deposition and vascular channels with staghorn pattern (B). Foci of necrosis and peritheliomatosis (C) and focal marked pleomorphism are seen (D). Resected large browny well-defined solitary fibrous tumor of the liver (E). Immunostaining of lesion (F-H); for Bcl2 (F), CD34 (G) and CD99 (H) shows positive results.

leiomyoma or lesions such as sclerosing hemangioma, and inflammatory pseudo tumor $(4,12)$.

Due to variable and non-specific radiologic findings and lots of overlaps in imaging features, precise radiologic diagnosis of SFTL is not possible and pathology should be considered for a definite diagnosis.

In histopathology examination, proliferation of ovoid spindle-like cells with pattern-less pattern architecture between collagen bundles and staghorn vessels are observed. CD34, bcl2 and CD99 are positive and S100, desmin and CD117 are negative in immunohistochemistry (6). Hyper cellularity, cytologic atypia, tumor necrosis, high mitotic activity (> 4/10 HPF) and infiltrative margins are demonstrated as malignant features by World Health Organization (WHO) (10).

Most SFTLs are categorized as benign tumors that have malignant potential. Surgical resection is considered as a main treatment. Chemotherapy and radiotherapy are recommended for cases with malignant potential or incomplete resection $(6,15)$. The follow-up often shows benign local recurrences. Nonetheless, there are few reports of metastatic spread to other areas of the body $(3,4)$ suggesting long-term follow up of these patients.

In conclusion, we report a rare case of hepatic solitary fibrous tumor with malignant transformation and review literature considering imaging features which could be valuable for radiologists. Although SFTLs are rare tumors, they should be considered as a differential diagnosis in patients with bulky well-defined hyper vascular mass that has a compression effect on surrounding structures. Though the radiologic features are non-specific, but hepatic solitary fibrous tumor should be considered in the differential diagnosis of hyper vascular liver masses with delayed contrast retention which did not fulfill imaging criteria of common hypervascular masses and findings in DWI could suggest malignant transformation. Histopathology and immunohistochemistry evaluations remain definite diagnostic tools and surgical resection is the treatment of choice. 


\section{Footnotes}

Authors' Contributions: Conception or design of the work: Niloofar Ayoobi Yazdi; Data collection: Niloofar Ayoobi Yazdi and Habibollah Dashti; data analysis and interpretation: Ali Jafarian, Masoomeh Safaeiand, and Maryam Fotouhi; drafting the article: Faezeh Salahshour and Maryam Fotouhi; critical revision of the article: Faezeh Salahshour and Maryam Fotouhi; final approval of the version to be published: Ghazaleh Amjad.

Conflict of Interests: The authors declare that there are no conflict of interests.

Funding/Support: This study was not funded by any funding source.

Informed Consent: Informed consent was obtained from the patient included in the report and the study protocol conforms to the ethical guidelines of the 1975 Declaration of Helsinki and Institutional Ethics Committee.

\section{References}

1. Klemperer PRCB. Primary neoplasms of the pleura. Arch. Pathol. 1931;11:385-412.

2. Ha JK, Park BJ, Kim YH, Lim YJ. Orbital solitary fibrous tumor : A case report and diagnostic clues.J Korean Neurosurg Soc. 2009;46(1):77-80. doi: 10.3340/jkns.2009.46.1.77. [PubMed: 19707501]. [PubMed Central: PMC2729832].

3. Yugawa K, Yoshizumi T, Mano Y, Kurihara T, Yoshiya S, Takeishi K, et al. Solitary fibrous tumor in the liver: Case report and literature review. Surg Case Rep. 2019;5(1):68. doi: 10.1186/s40792-019-0625-6. [PubMed: 31020464]. [PubMed Central: PMC6482201].

4. Degnan AJ, Lee KK, Minervini MI, Borhani AA. Metastatic extrapleural malignant solitary fibrous tumor presenting with hypoglycemia (Doege-Potter syndrome). Radiol Case Rep. 2017;12(1):113-9. doi: 10.1016/j.radcr.2016.10.014. [PubMed: 28228892]. [PubMed Central: PMC5310265].

5. Iglesias P, Diez JJ. Management of endocrine disease: A clinical update on tumor-induced hypoglycemia. Eur J Endocrinol. 2014;170(4):R14757. doi: 10.1530/EJE-13-1012. [PubMed: 24459236].

6. DeVito N, Henderson E, Han G, Reed D, Bui MM, Lavey R, et al. Clinical characteristics and outcomes for solitary fibrous tumor (SFT):
A single center experience. PLoS One. 2015;10(10). e0140362. doi 10.1371/journal.pone.0140362. [PubMed: 26469269]. [PubMed Central: PMC4607370].

7. Chen N, Slater K. Solitary fibrous tumour of the liver-report on metastasis and local recurrence of a malignant case and review of literature. World J Surg Oncol. 2017;15(1):27. doi: 10.1186/s12957-017-1102-y. [PubMed: 28100235]. [PubMed Central: PMC5242002].

8. Kueht M, Masand P, Rana A, Cotton R, Goss J. Concurrent hepatic hemangioma and solitary fibrous tumor: Diagnosis and management. J Surg Case Rep. 2015;2015(7). doi: 10.1093/jscr/rjv089. [PubMed: 26209579]. [PubMed Central: PMC4513232].

9. Silvanto A, Karanjia ND, Bagwan IN. Primary hepatic solitary fibrous tumor with histologically benign and malignant areas. Hepatobiliary Pancreat Dis Int. 2015;14(6):665-8. doi: 10.1016/s1499-3872(15)60365-4 [PubMed: 26663016].

10. Esteves C, Maia T, Lopes JM, Pimenta M. Malignant solitary fibrous tumor of the liver: AIRP best cases in radiologic-pathologic correlation. Radiographics. 2017;37(7):2018-25. doi: 10.1148/rg.2017160200. [PubMed: 29131777].

11. Fuksbrumer MS, Klimstra D, Panicek DM. Solitary fibrous tumor of the liver: Imaging findings. AJR Am J Roentgenol. 2000;175(6):1683-7. doi: 10.2214/ajr.175.6.1751683. [PubMed: 11090404].

12. Ginat DT, Bokhari A, Bhatt S, Dogra V. Imaging features of solitary fibrous tumors. AJR Am J Roentgenol. 2011;196(3):487-95. doi: 10.2214/AJR.10.4948. [PubMed: 21343490]

13. Yilmaz S, Kirimlioglu V, Ertas E, Hilmioglu F, Yildirim B, Katz D, et al Giant solitary fibrous tumor of the liver with metastasis to the skeletal system successfully treated with trisegmentectomy. Dig Dis Sci. 2000;45(1):168-74. doi: 10.1023/a:1005438116772. [PubMed: 10695631].

14. Sun K, Lu JJ, Teng XD, Ying LX, Wei JF. Solitary fibrous tumor of the liver: A case report. World J Surg Oncol. 2011;9:37. doi: 10.1186/1477-7819-9-37. [PubMed: 21443810]. [PubMed Central: PMC3079681].

15. Korkolis DP, Apostolaki K, Aggeli C, Plataniotis G, Gontikakis E, Volanaki D, et al. Solitary fibrous tumor of the liver expressing CD34 and vimentin: A case report. World J Gastroenterol. 2008;14(40):62614. doi: 10.3748/wjg.14.6261. [PubMed: 18985821]. [PubMed Central: PMC2761592].

16. England DM, Hochholzer L, McCarthy MJ. Localized benign and malignant fibrous tumors of the pleura. A clinicopathologic review of 223 cases. Am J Surg Pathol. 1989;13(8):640-58. doi: 10.1097/00000478198908000-00003. [PubMed: 2665534].

17. Inaoka T, Takahashi K, Miyokawa N, Ohsaki Y, Aburano T. Solitary fibrous tumor of the pleura: Apparent diffusion coefficient (ADC) value and ADC map to predict malignant transformation. J Magn Reson Imaging. 2007;26(1):155-8. doi: 10.1002/jmri.20942. [PubMed: 17659560]. 